\title{
Coulomb and Hard Core Skyrmion Tails
}

\author{
E. H. Rezayi ${ }^{1}$ and S. L. Sondhi ${ }^{2}$. \\ ${ }^{1}$ Department of Physics, California State University Los Angeles, CA 90032, USA \\ ${ }^{2}$ Department of Physics, Princeton University, Princeton, NJ 08544, USA
}

(January 6, 2018)

\begin{abstract}
Quantum Hall skyrmions are quantized solitons of a ferromagnetic $O(3)$ $\sigma$-model. The reference, classical, solutions depend upon the interaction between the electrons and exhibit completely different asymptotic profiles for the physical Coulomb interaction than for the hard core interaction frequently used to generate variational wavefunctions. In this note we show, by means of numerical calculations on (large) finite size systems at $\nu=1$, that this physically important difference, crucial for a sharp definition of their statistics, persists for the quantized skyrmions at $\nu=1$.
\end{abstract}

Typeset using REVTEX 
Theoretical and experimental work over the last few years has adduced strong evidence that the physics of the quantum Hall (QH) system in the vicinity of (filling factor) $\nu=1$ is best understood in terms of a ferromagnetic ground state right at commensuration which supports charged excitations with non-trivial spin topology christened "skyrmions" [1] [3]. The skyrmions arise as quantized solitons in a two dimensional $O(3) \sigma$-model which describes the low energy physics of the $\nu=1$ state, and mutatis mutandis of other ferromagnetic QH states. Very general considerations of homotopy suggest the possibility of topologically stable and non-trivial classical solitons in any two dimensional $O(3) \sigma$-model [4] but the issue of their dynamical stability is more complicated. As was first noted by Belavin and Polyakov [5], the leading (gradient squared) term in their energy functional is scale invariant in two dimensions; hence the explicit solutions obtained by them exhibit an arbitrary scale parameter and algebraic asymptotic profiles. Consequently, dynamical stability, i.e. the existence of a well defined size for the skyrmions, depends crucially on the sub-leading terms which are system specific.

In the theory of the $\nu=1 \mathrm{QH}$ this issue can be resolved in two very different ways. For the physical, Coulomb, interaction, the effective $\sigma$-model written in terms of the order parameter field $\mathbf{n}(\mathbf{r})$ is modified to [1]

$$
\mathcal{L}_{\text {eff }}=\frac{1}{2} \bar{\rho} \mathcal{A}(\mathbf{n}) \cdot \partial_{t} \mathbf{n}-\frac{1}{2} \rho^{s}(\nabla \mathbf{n})^{2}+\frac{1}{2} g \bar{\rho} \mu_{B} \mathbf{n} \cdot \mathbf{B}-\frac{e^{2}}{2 \epsilon} \int d^{2} r^{\prime} \frac{q(\mathbf{r}) q\left(\mathbf{r}^{\prime}\right)}{\left|\mathbf{r}-\mathbf{r}^{\prime}\right|},
$$

neglecting a Hopf term that does not affect the structure of the skyrmions. Here $\mathcal{A}$ is the vector potential of a unit magnetic monopole, $\rho^{s}$ the spin stiffness $\left(\rho^{s}=e^{2} /(16 \sqrt{2 \pi} \epsilon \ell)\right.$ for $\nu=1), \epsilon$ the semiconductor dielectric constant, $\ell$ the magnetic length and $q(\mathbf{r})=$ $\mathbf{n} \cdot\left(\partial_{x} \mathbf{n} \times \partial_{y} \mathbf{n}\right) / 4 \pi$ is the topological density of the spin field. The Zeeman (third) and Coulomb (fourth) terms in $\mathcal{L}_{\text {eff }}$ break the spatial scale invariance of the gradient (second) term and their competition then sets the size and energy of the skyrmions which now depend on the dimensionless ratio $\tilde{g}=\left(g \mu_{B} B\right) /\left(e^{2} / \epsilon \ell^{2}\right)$ of the Zeeman energy to the Coulomb energy. On the other hand, one can study a "hard core" interaction, consisting of a delta function projected to the lowest Landau level, which is a standard device in QH theory to generate 
variational wavefunctions [6]. Remarkably, the effective $\sigma$-model for this problem contains exactly the first three terms, i.e. it supports skyrmions of arbitrary size $\lambda$ at $g \mu_{B} B=0$ given by the Belavin/Polyakov solutions:

$$
n_{x}=\sqrt{1-f^{2}(r)} \cos (\theta), \quad n_{y}=\sqrt{1-f^{2}(r)} \sin (\theta), \quad n_{z}=f(r),
$$

where $f(r)=\left[(r / \lambda)^{2}-4\right] /\left[(r / \lambda)^{2}+4\right]\left[[7]\right.$. For $g \mu_{B} B \neq 0$, the stable solution has vanishing size. Despite this last pathology of the hard core problem, it would appear that its solutions could again be used for a variational solution of the Coulomb problem, i.e. by optimizing $\lambda$.

It is a fact of some consequence, that this cannot be done. It turns out [1, 8 , that the skyrmion profile in Eq (2) decays too slowly $\left(n^{z}-1 \sim(\lambda / r)^{2}\right)$ to yield a finite answer for the Zeeman energy, necessitating a calculation of its true asymptotic form in the Coulomb problem which can be shown to go as $n^{z}-1 \sim e^{-r / \sqrt{g}}$, i.e. the Coulomb skyrmion has a much shorter tail than the hard core variant. Clearly, if this was not taken into account one would falsely conclude that Coulomb skyrmions are always microscopic. Further, it was shown by Yang and Sondhi [9] that the statistics of the skyrmions is ill-defined in hard core multi-skyrmion states precisely on account of the long tails of the skyrmions but well defined for the shorter tailed Coulomb versions. Finally, the difference in asymptotic behavior was shown recently to lead to binding between singly charged skyrmions at small Zeeman energies, albeit at unphysically small values [10].

The above considerations were all in the framework of classical solutions of the $\sigma$-model and the closely related Hartree-Fock approximation [2]. The corresponding quantum states require inclusion of fluctuations that quantize spin and angular momentum [11,12, and restore the symmetries broken in the classical solutions, and one may wonder if the distinction between the tails of hard core and Coulomb skyrmions survives when this is done. In this note we report numerical calculations on the smallest skyrmions, i.e. those with one extra reversed spin for which the fluctuations are the most severe, and show that this important distinction is indeed preserved.

Results: We study a QH system on a sphere [6] at one flux quantum less than the finite 
size version of $\nu=1$, i.e. with $N_{\phi}$ flux quanta passing through the surface of the sphere and $N=N_{\phi} \equiv 2 K$ particles. This is the one quasielectron sector and its states are related to those in the one quasihole sector $\left(N=N_{\phi}+2\right)$ by a particle-hole transformation. The spectrum in this sector has a set of states with equal values of the angular momentum $L$ and spin $S$. The states with $L=S=N / 2-1$ are the multiplet of the fully polarized quasielectron (it has just one reversed spin) and those with lower $L, S$ are skyrmions of various sizes culminating in the infinite skyrmion (it has the size of the system) with $L=S=0$. We have studied the smallest of the skyrmion states, i.e. with $L=S=N / 2-2$ and maximum $L_{z}$ for simplicity, for system sizes up to 300 electrons for both hard core and Coulomb interactions.

For the hard core interaction, an exact wavefunction can be written down. In second quantized notation this takes the form,

$$
|\Psi\rangle=\frac{1}{\sqrt{A}} \sum_{m}\left\{\frac{1}{\sqrt{2 K}} c_{m \uparrow} c_{m \downarrow}^{\dagger} c_{K-1, \downarrow}^{\dagger}+\sqrt{\frac{K+m}{K-m}} c_{m \uparrow} c_{m-1 \downarrow}^{\dagger} c_{K, \downarrow}^{\dagger}\right\}\left|\nu_{\uparrow}=1\right\rangle
$$

where $A=(2 K+1) \sum_{n=2}^{2 K} 1 / n \approx(2 K+1)(\ln (2 K)+\gamma-1)$, and $\gamma$ is the Euler's constant. From this we can easily compute the occupation of various orbitals by the down spins,

$$
\begin{aligned}
n(K) & =1-\left(1-1 / N_{\phi}\right) / A \\
n(K-1) & =N_{\phi}\left(1-1 / N_{\phi}\right) / A \\
\vdots & \\
n(K-m) & =\left(N_{\phi}+1\right) /(m A)-\left(1-1 / N_{\phi}\right) / A \quad m \geq 2 .
\end{aligned}
$$

It is clear from these formulas that one of the down spins is localized at the north pole (top orbital). For $\mathrm{N}=300$, in Fig 1 we plot the spatial density for the other down spin $\sigma_{\downarrow}(r)=\frac{1}{R^{2}} \sum_{m=-K}^{K-1}\left|\psi_{m}(\hat{\Omega})\right|^{2} n_{\downarrow}(m)$ by excluding the $m=K$ orbital. Here $r=2 R \sin \theta / 2$ is the chord distance, $R=\sqrt{K} \ell$ is the radius of the sphere. Evidently, $\sigma_{\downarrow}(r)$ is peaked near the nominal location of the quasielectron. In the same figure we plot the result for the exact wavefunction for Coulomb interactions which was obtained by numerical diagonalization. One can see a considerable difference in the degree of localization of the second reversed spin 
between these two potentials. The difference in the tails, however, may not be immediately apparent from this plot. It becomes apparent when we plot the data on a double-logarithmic scale in Fig 2, where it is clearly seen that the down spins are strongly localized in the Coulomb case when compared with the hard core wavefunction; at the opposite pole there are seven orders of magnitude separating the two $\operatorname{results}\left(3.1 \times 10^{-14}\right.$, vs. $\left.8.5 \times 10^{-6}\right)$ ! Moreover, the hard core distribution follows the $1 / r^{2}$ decay expected from the sigma model analysis while the Coulomb result decays faster than any power consistent with the predicted exponential behavior. The asymptotic behavior on the sphere for the hard core potential to leading order in system size can easily shown to be $\frac{N_{\phi}+1}{4 \pi \ell^{2} A}\left(\frac{4}{(r / \ell)^{2}}-\frac{1}{K}\right)$, see the dashed line in Fig 1. Yet another probe of this difference is the large system behavior of the down spin occupation of a given orbital near the location of the quasielectron. The hard core wavefunction is easily seen to lead to a logarithmic vanishing of this quantity in the large system limit which implies that the quasielectron is not a well defined object at infinite system size: one of its reversed spins is spread over the entire system. By contrast the Coulomb quasielectron is well defined, examination of the variation of the occupation with system size displays clear evidence of finiteness in the large system limit (Fig 3). For comparison, we have produced a similar plot for the hard-core potential in Fig 4. Only the occupation of the top orbital shows scaling with system size consistent with its being localized. Others show noticeable deviations as expected for delocalization of the second reversed spin.

In summary, there is a crucial difference in the asymptotic behavior of hard core (scale invariant $\sigma$-model) skyrmions and Coulombic skyrmions which is present also in the fully quantized and symmetric many-body states of the lowest Landau level problem. Hard core skyrmions have algebraic profiles and are ill-defined in the large system limit while Coulomb skyrmions have exponential profiles and have a definite size. This difference is crucial to the energetic stability of Coulomb skyrmions, their possessing a well defined statistics and to their binding at extremely small Zeeman energies. Two remarks are in order. First, the distinction we have drawn here between the skyrmions of the hard-core and the Coulomb 
potentials trivially vanishes for infinite skyrmions as they have no tails. Second, any generic perturbation of the hard core interaction (one which produces a direct interaction in addition to the exchange) will break the scale invariance and give a well defined size to the skyrmions; the hard core limit, though important in that it enables an exact analysis, is non-generic in this regard.

\section{ACKNOWLEDGMENTS}

We are grateful to A. Karlhede, S. A. Kivelson and K. Yang for useful discussions. This work was supported in part by NSF grant No. DMR-9632690 and the A. P. Sloan Foundation (SLS) and NSF grant No. DMR-9420560 (EHR). 


\section{REFERENCES}

[1] S. L. Sondhi, A. Karlhede, S. A. Kivelson and E. H. Rezayi, Phys. Rev. B 47, 16419 (1993). See also, E. H. Rezayi, Phys. Rev. B 36, 5454 (1987) and 43, 5944 (1991); D.-H. Lee and C. L. Kane, Phys. Rev. Lett. 64, 1313 (1990).

[2] H. A. Fertig, L. Brey, R. Côté and A. H. MacDonald, Phys. Rev. B 50, 11018 (1994; H. A. Fertig et al., Phys. Rev. B 55, 10671 (1997).

[3] S. E. Barrett, G. Dabbagh, L. N. Pfeiffer, K. W. West and R. Tycko, Phys. Rev. Lett. 74, 5112 (1995); A. Schmeller, J. P. Eisenstein, L.N. Pfeiffer and K.W. West, Phys. Rev. Lett. 75, 4290 (1995); E. H. Aifer, B. B. Goldberg and D. A. Broido, Phys. Rev. Lett. 76, 680 (1996); D. K. Maude et. al., Phys. Rev. Lett. 77, 4604 (1996).

[4] For a review see R. Rajaraman, Solitons and Instantons (North-Holland, Amsterdam, 1982).

[5] A. A. Belavin and A. M. Polyakov, JETP Lett. 22, 245 (1975).

[6] F. D. M. Haldane, Phys. Rev. Lett. 51, 645 (1983); see also S. Trugman and S. Kivelson, Phys. Rev. B 31, 5280 (1985).

[7] This conclusion followed from the work of one of us (EHR) in [1] that showed that the excitations of the hard core model were degenerate in energy at different sizes; we do not know of a constructive proof of this at present. This connection was noted in the context of the Hartree-Fock approximation in [2].

[8] K. Lejnell, "A Long Wavelength Study of Quantum Hall Skyrmions", Thesis (Stockholm University, 1996); K. Lejnell, A. Karlhede and S. L. Sondhi, "Effective Action Studies of Quantum Hall Skyrmions", (in preparation).

[9] K. Yang and S. L. Sondhi, Phys. Rev. B 54, 2331 (1996).

[10] D. Lilliehook, K. Lejnell, A. Karlhede and S. L. Sondhi, Phys. Rev. 56, 6805 (1997). 
[11] C. Nayak and F. Wilczek, Phys. Rev. Lett. 77, 4418 (1996).

[12] A. H. MacDonald, H. A. Fertig and L. Brey, Phys. Rev. Lett. 76, 2153 (1996). 


\section{FIGURES}

FIG. 1. The spatial distribution of the second reversed spin for the Coulomb and the hard core potential for a 300-electron size systems. The dashed line is the asymptotic behavior to leading order in system size given in text. The inset shows the tails of the distribution.

FIG. 2. The log-log plot of Fig 1. The differences in the tail can be clearly seen. The density for the Coulomb tail is curved indicating it vanishes faster than any power. The straight line segment for hard core gives the $1 / r^{2}$ dependence.

FIG. 3. The plot of occupation amplitudes for the top 4 orbitals for the Coulomb potential for up to $\mathrm{N}=300$ plotted versus $1 / \mathrm{N}$. All are essentially independent of system size and indicate localization of the second reversed spin.

FIG. 4. Same as Fig 3 except for hard core potential plotted for comparison. Only the first reversed spin at the top orbital can be seen to be localized. The others show a noticeable decrease and ultimately vanish logarithmically with system size. 